\title{
The potential of XPO1 inhibitors as a game changer in relapsed/refractory hematologic malignancies
}

\author{
Henan Wang ${ }^{\mathrm{a}}$, Jing Yang ${ }^{\mathrm{a}}$, Liang Wang ${ }^{\mathrm{a},{ }^{*}}$ \\ ${ }^{a}$ Department of Hematology, Beijing Tongren Hospital, Capital Medical University, Beijing 100730, China.
}

\begin{abstract}
XP01 is a transporter receptor protein that transports leucine-rich proteins from the nucleus into the cytoplasm through the nuclear pore complex. In hematologic malignancies, XPO1 is often overexpressed, leading to abnormal regulation of cell growth and apoptosis or abnormal cell cycle regulation. Therefore, XPO1 inhibitors can be used as targeted drugs to block the transport of overexpressed XPO1, thus treating hematologic malignancies. We summarized the use of XPO1 inhibitors in clinical studies according to different hematologic malignancies and reviewed their efficacy and toxicity.
\end{abstract}

Keywords: XP01 inhibitor, hematologic malignancies, selinexor, targeted therapy

\section{Introduction}

The nucleus is an organelle that coats genetic material with a double membrane, separating transcription in the nucleus from translation in the cytoplasm. To achieve adequate cellular function, this spatial division in eukaryotic cells requires selective and efficient bidirectional transport of specific proteins and mRNAs through nuclear pore complexes (NPCs) in the nuclear membrane. The passage of macromolecular $(>40 \mathrm{kDa})$ cargo through NPCs requires specific transporter receptor proteins. The mammalian nuclear adhesin family is the main family of transporter receptor proteins, consisting of 20 members, including nuclear adhesin protein $\alpha$ 1-6, nuclear adhesin protein $\beta 1$, and exportin 1 (XPO1). Nuclear adhesins use the energy in the RanGTPase complex to insert or export them into or out of the nucleus, depending on the presence of precise transport signals, nuclear localization signals, or nuclear export signals (NES) in cargo proteins.

$\mathrm{XPO} 1$ is the main transporter receptor protein through which leucine-rich protein enters the cytoplasm from the nucleus via NPCs. In the nucleus, XPO1 binds to NES-

\footnotetext{
* Corresponding author: Liang Wang

Mailing address: Department of Hematology, Beijing Tongren Hospital, Capital Medical University, Beijing 100730, China.

E-mail: wangliangtrhos@126.com

Received: 06 May 2020 / Accepted: 03 June 2020
}

containing cargo and forms a ternary complex with RanGTP, passing through NPCs and entering the cytoplasm. The XPO1-cargo protein, which is driven by GTP hydrolysis, contains almost all tumor suppressor proteins (TSPs; such as p53, Rb, BRCA1/2, APC, and survivin), cell cycle regulatory proteins (such as p21, p27, and galactin-3), the glucocorticoid receptor (GR), and chemotherapy targets (such as DNA topoisomerase) [1-2].

XPO1 mutations and/or overexpression have been reported in almost all malignancies and have been associated with enhanced extranuclear cargo transport, resulting in apoptotic inactivation, disrupted cell cycle regulation, abnormal cell growth signaling, impaired glucocorticoid signaling, and chemotherapy resistance. For example, repeated mutations in the highly conserved region of XPO1 contribute to the development of chronic lymphocytic leukemia, while the overexpression of XPO1 is associated with solid tumors and hematologic malignancies [35]. XPO1 inhibitors are, therefore, of great clinical value in the treatment of malignant tumors. These inhibitors reversibly bind to XPO1 through covalent bonds with Cys528 residues in the tank, inactivating its nuclear transport function [6]. Studies have also shown that XPO1 inhibitors preferentially destroy the $3 \mathrm{D}$ nuclear tissue of telomeres in tumor cells [7], which may be another mechanism by which XPO1 inhibitors inhibit XPO1. The selective inhibitor of nuclear export compounds under study include KPT-185, KPT-251, KPT-276, KPT-335 (verdinexor), KPT-8602 (eltanexor), and KPT-330 (selinexor). Selinexor was approved on an accelerated basis by the US FDA in combination with dexamethasone for the 
treatment of relapsed/refractory multiple myeloma (MM) after four lines of treatment [8]. As a second generation oral XPO1 inhibitor, eltanexor has significantly reduced blood-brain barrier permeability compared with selinexor, thereby reducing adverse effects, such as central nervous system-mediated anorexia and weight loss, and may have a better safety profile and wider therapeutic window [9]. In the following sections, the clinical progress of XPO1 inhibitors in different hematologic malignancies is reviewed in detail.

\section{Use of XPO1 inhibitors in MM}

In view of the considerable effects of XPO1 inhibitors in preclinical application, multiple subsequent clinical studies have been conducted, and the use of XPO1 inhibitors in MM patients has also shown a good response. Selinexor has a certain activity in patients with extremely refractory MM, and combination therapy including selinexor has shown that it sensitizes MM to some drugs that were previously insensitive, showing a good characteristic of inducing re-sensitivity [10]. In addition, a second-generation XPO1 inhibitor, eltanexor, has gradually entered clinical studies and has shown considerable antitumor activity in patients with relapsed/refractory MM treated with endline therapy [11].

\section{Selinexor plus dexamethasone}

There are many combinations of drugs, among which selinexor combined with dexamethasone has been evaluated in many studies. A phase I study [12] demonstrated that the overall response rate (ORR) of 84 patients with RRMM and Waldenstrom macroglobulinemia treated with selinexor and dexamethasone was $10 \%$, including 1 patient with a complete response (CR) and 7 patients with a partial response (PR). The median response time was 1 month (1-3 months), and the median duration of response was 5 months (2-11 months). Minimal response (MR) was observed in an additional 13 patients (15\%), with a clinical benefit rate of $25 \%$. There are mainly three categories of MM drugs on the market in China: proteasome inhibitors, immunotherapy drugs (IMiDs), and CD38 monoclonal antibodies. Studies have shown that the median survival time of patients with MM that is refractory to all these drugs is only $1.3-3.5$ months, with a very poor prognosis. In a multicenter phase II study [13], 122 patients with RRMM who failed the above three categories of $\mathrm{MM}$ drugs received selinexor and dexamethasone, and $32(26 \%)$ had a partial or better response, including 2 patients $(2 \%)$ with a stringent CR, $6(5 \%)$ with a very good partial response (VGPR), and $24(20 \%)$ with a PR. The median progression-free survival (PFS) and overall survival (OS) were 3.7 and 8.6 months, respectively, demonstrating significant improvement in the prognosis of end-line treated MM patients.

XPO1 inhibitors plus proteasome inhibitors and dexamethasone
Preclinical studies have shown that XPO1 inhibitors have a synergistic effect with proteasome inhibitors and can induce MM re-sensitivity to proteasome inhibitors. In a phase I study [14], 18 patients with RRMM were treated with selinexor, ixazomib, and low-dose dexamethasone. Of the 14 evaluable patients, 2 had a VGPR; 1 had a PR; 7 had stable disease (SD); and 4 had progressive disease (PD), with the longest response duration of 14 months. In another study [15], 21 patients with RRMM received selinexor combined with carfilzomib and dexamethasone. The results showed $71 \%$ MR, 48\% PR, and 14\% VGPR. In addition, in a study in which 42 patients received bortezomib [16], an overall response was achieved in 25 (63\%) of the 40 evaluable patients, including 3 CRs, 9 VGPRs, and 13 PRs, with a median PFS of 9 months. The data from these studies confirmed the synergistic effect observed between selinexor and proteasome inhibitors and a better therapeutic effect, which provided a new therapeutic approach for patients with RRMM.

\section{XPO1 inhibitors plus IMiDs and dexamethasone}

Multiple previous studies and meta-analyses have shown that lenalidomide (a second generation IMiD) has a good synergistic effect with a variety of MM drugs, so a combination drug strategy based on lenalidomide is often recommended in patients with RRMM. Darrell J White et al [17] used selinexor in combination with lenalidomide and dexamethasone in eight patients who had been newly diagnosed with MM. The treatment was efficacious in six of the seven patients who were assessed for efficacy (ORR $86 \%$ ). In a previous study [18], 18 patients with RRMM received selinexor, lenalidomide, and dexamethasone. The ORR was $73 \%$ among the 15 patients who could be evaluated for efficacy, and the ORR was $91 \%$ in the lenalidomide-sensitive group $(n=11)$. Another study [19] used a combination of selinexor, pomalidomide (a third generation IMiD), and dexamethasone (SPd) in 48 patients with RRMM. The ORR was 58\% (7 cases of VGPR and 11 cases of PR), and the median PFS was 12.2 months in patients who were first treated with pomalidomide $(\mathrm{N}=31)$. In 13 patients with disease that was refractory to lenalidomide/pomalidomide, the ORR was $31 \%$ (4 patients with a PR), and the median PFS was 4.2 months. These studies indicate that for patients with RRMM, the all-oral SPd regimen could achieve a lasting effect, and it had a better remission rate and longer PFS in patients with MM that was sensitive to IMiDs.

\section{XPO1 inhibitors plus other classifications of drugs and dexamethasone}

The treatment of MM has entered an era of monoclonal antibodies, and the combined daratumumab-based regimen has achieved good results in patients with RRMM. A phase Ib study [20] used a combination of selinexor, daratumumab, and dexamethasone (SDd) in 25 patients with RRMM who were previously exposed to proteasome inhibitors and IMiDs. Among the 19 patients with daratumumab-sensitive disease, the ORR was $74 \%$ (5 
VGPRs, 9 PRs, 2 MRs, 2 with SD, and 1 with PD). In the two cases of daratumumab refractory MM, there was one case of PD and one case of SD. This study confirmed that SDd has great clinical application value in patients with disease that is refractory to proteasome inhibitors/IMiDs. In addition, studies [21] have combined liposomal doxorubicin with selinexor and dexamethasone in the treatment of RRMM. Among the 27 patients enrolled, the ORR was $15 \%$, and the clinical benefit rate (MR or better) was $26 \%$, suggesting that adding anthracyclines to the combination of XPO1 inhibitors and dexamethasone does not seem to further enhance efficacy.

These studies indicate that a combination drug strategy based on an XPO1 inhibitor provides new treatment options for patients with RRMM and significantly improved the prognosis of patients with pandrug-resistant MM. Currently, clinical trials of XPO1 inhibitors are being conducted in patients newly diagnosed with MM.

\section{The toxicity profiles of XPO1 inhibitors}

Common adverse reactions in patients treated with XPO1 inhibitors are gastrointestinal tract reactions (anorexia, nausea, vomiting, diarrhea, and constipation), hematologic adverse reactions (thrombocytopenia, anemia, neutropenia, and lymphopenia), mental status changes, fatigue, dizziness, insomnia, pneumonia, liver function abnormalities, and electrolyte disorders. Gastrointestinal adverse reactions can be alleviated by prophylactic megestrol acetate and ondansetron, and symptoms can be alleviated by reductions in the dosage or discontinuation of medication in patients with more severe adverse reactions. A second generation oral XPO1 inhibitor, eltanexor, has significantly lower blood-brain barrier permeability than selinexor, and is therefore superior to selinexor in central nervous system-mediated adverse effects, such as anorexia and weight loss.

\section{Clinical trials of XPO1 inhibitors in the treat- ment of acute myeloid leukemia (AML)}

\section{XPO1 inhibitor monotherapy in AML}

XPO1 inhibitor monotherapy showed some anti-leukemia activity in AML patients. A dose-escalation study [22] included 95 patients with recurrent or refractory AML. Of the 81 evaluable patients, $11(14 \%)$ achieved objective remission (OR), including 5 patients with a $C R$ and 2 patients with a $\mathrm{CR}$ and incomplete peripheral blood cell count recovery (CRi). The median PFS (5.1 versus 1.3 months) and OS (9.7 versus 2.7 months) showed significant improvements in responders compared to non-responders. The results of this study indicated that selinexor is safe and effective as monotherapy for patients with recurrent or refractory AML.

\section{XPO1 inhibitor-based combination therapy in AML}

Combination therapy with an XPO1 inhibitor showed bet- ter disease control than monotherapy. A study [23] combined selinexor with decitabine in 25 patients with refractory/relapsed AML $(n=20)$ or previously untreated older adults $(>60$ years old $)(n=5)$. Ten patients responded to treatment $(40 \%)$, and of the five elderly patients, four responded to treatment. The PFS and OS for patients were 11.8 and 12.9 months, respectively, for responders compared with 4.4 and 5.9 months, respectively, for nonresponders. This study demonstrated that the combination of selinexor and decitabine resulted in a higher ORR, and this combination significantly improved the therapeutic response, especially in newly diagnosed elderly patients. In a study of the combination of selinexor and sorafenib, a significant apoptosis-inducing effect was also found, and patients with FLT3 inhibitor-resistant disease also had a better CR rate (45\%) [24]. There are many studies on selinexor combined with cytarabine and other drugs. In one study, 20 patients with newly diagnosed or recurrent/ refractory AML were treated with selinexor combined with high dose cytarabine and mitoxantrone [25]. There were 10 cases $(50 \%)$ of CR, 3 cases $(15 \%)$ of $\mathrm{CRi}, 1$ case $(5 \%)$ of PR, and 6 cases $(30 \%)$ of PD, and there was an ORR of $70 \%$. Sweet, K et al treated 21 high-risk AML patients with selinexor in combination with daunorubicin and cytarabine $(7+3$ regimen $)$. Of the 19 evaluable patients, $10(53 \%)$ achieved a CR/CRi. There was no doserestricted toxicity during induction. This study confirmed a synergistic effect between selinexor and daunorubicin, and $80 \mathrm{mg}$ of selinexor can be safely used in combination with a $7+3$ induction regimen to treat patients with AML. Timothy $S$ et al [26] applied a $7+3$ regimen combined with selinexor in elderly patients over 60 years old, and all patients taking selinexor achieved a clinical response. Another study [27] used selinexor in combination with the CLAG regimen to treat 40 patients with recurrent or refractory AML, and 18 patients (45\%) achieved a CR or CRi, with a median remission period of 9.1 months, median PFS of 6.1 months, and OS of 6.1 months. This study confirmed that selinexor-based combination therapy in the treatment of patients with recurrent AML provided an effective and reasonable alternative for bridging transplantation.

\section{Use of XPO1 inhibitors in non-Hodgkin lym- phoma (NHL)}

In a preclinical study, Muqbil, I et al [28] combined selinexor with dexamethasone in an animal model of NHL and observed reduced expression of caspase- 3 and significant reduction in XPO1 in the combined treatment group. In addition, studies have revealed that [29] selinexor can enable ibrutinib-resistant mantle cell lymphoma (MCL) to overcome drug resistance, which provides a new therapeutic approach for patients with ibrutinib-resistant MCL. In clinical studies, XPO1 inhibitors have demonstrated long-lasting and effective antitumor activity. A phase I study [30] included 79 NHL patients, 47 of whom were 
in the dose escalation group, who received $3-80 \mathrm{mg} / \mathrm{m}^{2}$ selinexor for 3 or 4 weeks. A total of 32 patients in the extended cohort received either $35 \mathrm{mg} / \mathrm{m}^{2}$ or $60 \mathrm{mg} / \mathrm{m}^{2}$ selinexor. Of the 70 evaluable patients, 22 (31\%) achieved OR, including 4 CRs and 18 PRs. SD was obtained in 21 cases $(30 \%)$, and the disease control rate was $61 \%$. These results suggest that oral administration of $35 \mathrm{mg} / \mathrm{m}^{2}$ selinexor is a safe treatment and has considerable anticancer activity in patients with relapsed/refractory NHL. A phase $2 \mathrm{~b}$ study conducted in patients with DLBCL included 110 patients [31] treated with $60 \mathrm{mg}$ selinexor twice weekly on a 28-day cycle. In the initial interim analysis of 32 patients, the ORR was $34.4 \%$ (5 CRs and 6 PRs). The median duration of response was 8.4 months, of which some CRs lasted longer than 24 months, showing a profound and lasting response. Another study used the same medication regimen to treat 129 relapsed/refractory patients with DLBCL who had previously received 2-5 lines of therapy, and this regimen achieved an ORR of $27.6 \%$ (14 CRs and 21 PRs). These results suggest the potential of selinexor as a new therapy for DLBCL. The US FDA has included the use of selinexor for treating patients with relapsed/refractory DLBCL who have received at least two lines of treatment for accelerated approval. In addition, an isolated CNS relapse was reported [32] in a patient with DLBCL, who was then given $60 \mathrm{mg}$ oral selinexor twice a week (day 1 and day 3). After five months of selinexor treatment, the patient's symptoms disappeared, and an MRI scan showed that the brain tumor had completely disappeared. This report demonstrates the blood-brain barrier permeability of selinexor and its significant efficacy in the treatment of central nervous system DLBCL.

\section{Conclusions}

As new, targeted drugs, XPO1 inhibitors have shown good antitumor activity in a variety of hematologic malignancies. Increasing clinical studies on XPO1 inhibitors are gradually being conducted. Selinexor, a first-generation XPO1 inhibitor, was approved for use in RRMM and is about to be approved for treatment of relapsed/refractory DLBCL. Second generation XPO1 inhibitors provide a wider therapeutic window, and their lower blood-brain barrier permeability supports a continuous medication approach, which will further improve the efficacy and safety of treatment. In conclusion, the discovery of XPO1 inhibitors has provided new therapeutic options and may be a potential game changer for relapsed/refractory hematologic malignancies.

\section{References}

1. Gravina G L, Senapedis W, McCauley D, et al. Nucleocytoplasmic transport as a therapeutic target of cancer. Journal of hematology \& oncology, 2014, 7(1): 85.

2. Conforti F, Wang Y, Rodriguez J A, et al. Molecular path- ways: anticancer activity by inhibition of nucleocytoplasmic shuttling. Clinical Cancer Research, 2015, 21(20): 4508-4513.

3. Kuruvilla J, Savona M, Baz R, et al. Selective inhibition of nuclear export with selinexor in patients with nonHodgkin lymphoma. Blood, The Journal of the American Society of Hematology, 2017, 129(24): 3175-3183.

4. Noske A, Weichert W, Niesporek S, et al. Expression of the nuclear export protein chromosomal region maintenance/exportin 1/Xpo1 is a prognostic factor in human ovarian cancer. Cancer: Interdisciplinary International Journal of the American Cancer Society, 2008, 112(8): 1733-1743.

5. Shen A, Wang Y, Zhao Y, et al. Expression of CRM1 in human gliomas and its significance in p27 expression and clinical prognosis. Neurosurgery, 2009, 65(1): 153-160.

6. Burke R T, Marcus J M, Orth J D. Inhibition of exportin-1 function results in rapid cell cycle-associated DNA damage in cancer cells. Oncotarget, 2017, 8(24): 39460.

7. Taylor-Kashton C, Lichtensztejn D, Baloglu E, et al. XPO1 inhibition preferentially disrupts the 3D nuclear organization of telomeres in tumor cells. Journal of cellular physiology, 2016, 231(12): 2711-2719.

8. XP01 Inhibitor Approved for Multiple Myeloma. Cancer Discovery, 2019, 9(9): 1150-1151

9. Etchin J, Berezovskaya A, Conway A S, et al. KPT-8602, a second-generation inhibitor of XP01-mediated nuclear export, is well tolerated and highly active against AML blasts and leukemia-initiating cells. Leukemia, 2017, 31(1): 143-150.

10. Peterson T J, Orozco J, Buege M. Selinexor: A First-inClass Nuclear Export Inhibitor for Management of Multiply Relapsed Multiple Myeloma. Annals of Pharmacotherapy, 2020, 54(6): 577-582.

11. Cornell R F, Rossi A C, Baz R, et al. Eltanexor (KPT-8602), a second-generation selective inhibitor of nuclear export (SINE) compound, in patients with refractory multiple myeloma. Blood, 2017, 130(Suppl 1): 3134-3134.

12. Chen C, Siegel D, Gutierrez M, et al. Safety and efficacy of selinexor in relapsed or refractory multiple myeloma and Waldenstrom macroglobulinemia. Blood, The Journal of the American Society of Hematology, 2018, 131(8): 855-863.

13. Chari A, Vogl D T, Gavriatopoulou M, et al. Oral SelinexorDexamethasone for Triple-Class Refractory Multiple Myeloma. New England Journal of Medicine, 2019, 381(8): 727-738.

14. Salcedo M, Lendvai N, Mastey D, et al. Phase I Study of Selinexor, Ixazomib, and Low-dose Dexamethasone in Patients With Relapsed or Refractory Multiple Myeloma. Clinical Lymphoma, Myeloma and Leukemia, 2020, 20(3): 198-200.

15. Jakubowiak A J, Jasielec J K, Rosenbaum C A, et al. Phase 1 study of selinexor plus carfilzomib and dexamethasone for the treatment of relapsed/refractory multiple myeloma. British journal of haematology, 2019, 186(4): 549-560.

16. Bahlis N J, Sutherland H, White D, et al. Selinexor plus 
low-dose bortezomib and dexamethasone for patients with relapsed or refractory multiple myeloma. Blood, 2018, 132(24): 2546-2554.

17. White D J, Lentzsch S, Gasparetto C, et al. Safety and Efficacy of the Combination of Selinexor, Lenalidomide and Dexamethasone (SRd) in Patients with Newly Diagnosed Multiple Myeloma. 2019.

18. White D J, Bahlis N J, Venner C P, et al. A phase Ib/II trial of selinexor combined with lenalidomide and low dose dexamethasone in patients with relapsed/refractory multiple myeloma. Blood, 2017, 130(Supplement 1): 1861-1861.

19. Chen C I, Bahlis N, Gasparetto C, et al. Selinexor, Pomalidomide, and Dexamethasone (SPd) in Patients with Relapsed or Refractory Multiple Myeloma. 2019.

20. Gasparetto C J, Lentzsch S, Schiller G J, et al. Deep and durable responses with selinexor, daratumumab, and dexamethasome (SDd) in patients with multiple myeloma (MM) previously exposed to proteasome inhibitors and immunomodulatory drugs: results of phase $1 \mathrm{~b}$ study of SDd. Blood, 2018, 132(Supplement 1): 599-599.

21. Baz R, Zonder J A, Shain K H, et al. Phase I/II study of liposomal doxorubicin (DOX) in combination with selinexor (SEL) and dexamethasone (DEX) for relapsed and refractory multiple myeloma (RRMM). Blood, 2017, 130(Supplement 1): 3095-3095.

22. Garzon R, Savona M, Baz R, et al. A phase 1 clinical trial of single-agent selinexor in acute myeloid leukemia. Blood, The Journal of the American Society of Hematology, 2017, 129(24): 3165-3174.

23. Bhatnagar B, Zhao Q, Mims A S, et al. Selinexor in combination with decitabine in patients with acute myeloid leukemia: results from a phase 1 study. Leukemia \& Lymphoma, 2020, 61(2): 387-396.

24. Daver N G, Assi R, Kantarjian H M, et al. Final results of phase I/II study of selinexor (SEL) with sorafenib in patients (pts) with relapsed and/or refractory (R/R) FLT3 mutated acute myeloid leukemia (AML). Blood, 2018, 132(Supplement 1): 1441-1441.

25. Wang A Y, Weiner H, Green M, et al. A phase I study of selinexor in combination with high-dose cytarabine and mitoxantrone for remission induction in patients with acute myeloid leukemia. Journal of hematology \& oncology, 2018, 11(1): 4.

26. Pardee T S, Wood K C, Lin K H, et al. Selinexor in Combination with Induction and Consolidation Therapy in Older Adults with AML Is Highly Active. 2019.

27. Abboud R, Chendamarai E, Rettig M P, et al. Selinexor combined with cladribine, cytarabine, and filgrastim in relapsed or refractory acute myeloid leukemia. Haematologica, 2019.

28. Muqbil I, Aboukameel A, Elloul S, et al. Anti-tumor activity of selective inhibitor of nuclear export (SINE) compounds, is enhanced in non-Hodgkin lymphoma through combination with mTOR inhibitor and dexamethasone. Cancer letters, 2016, 383(2): 309-317.

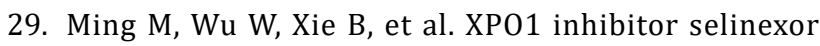
overcomes intrinsic ibrutinib resistance in mantle cell lymphoma via nuclear retention of IкB. Molecular cancer therapeutics, 2018, 17(12): 2564-2574.

30. Kuruvilla J, Savona M, Baz R, et al. Selective inhibition of nuclear export with selinexor in patients with nonHodgkin lymphoma. Blood, The Journal of the American Society of Hematology, 2017, 129(24): 3175-3183.

31. Maerevoet M, Vermaat J, Canales M A, et al. Single Agent Oral Selinexor Demonstrates Deep and Durable Responses in Relapsed/Refractory Diffuse Large B-Cell Lymphoma (DLBCL) in Both GCB and Non-GCB Subtypes: The Phase 2b Sadal Study. Blood, 2018, 132(Supplement 1): 1677-1677.

32. Bobillo S, Abrisqueta P, Carpio C, et al. Promising activity of selinexor in the treatment of a patient with refractory diffuse large B-cell lymphoma and central nervous system involvement. haematologica, 2018, 103(2): e92.

Cite this article as: Wang H, Yang J, Wang L. The potential of XPO1 inhibitors as a game changer in relapsed/ refractory hematologic malignancies[J]. Aging Pathobiology and Therapeutics, 2020, 2(2): 109-113. 